Article

\title{
Steroidogenic Factor 1 (NR5A1) Activates ATF3 Transcriptional Activity
}

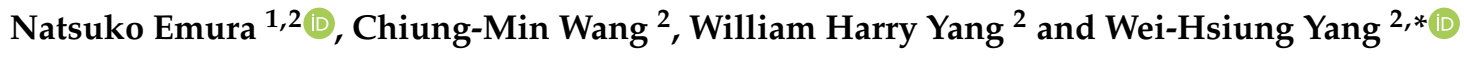 \\ 1 The United Graduate School of Agricultural Sciences, Iwate University, Morioka, Iwate 020-8550, Japan; \\ u3118002@iwate-u.ac.jp \\ 2 Department of Biomedical Sciences, Mercer University School of Medicine, Savannah, GA 31404, USA; \\ meowy200@yahoo.com (C.-M.W.); meowy100@yahoo.com (W.H.Y.) \\ * Correspondence: yang_w@mercer.edu; Tel.: +1-912-721-8203; Fax: +1-912-721-8268
}

Received: 31 January 2020; Accepted: 18 February 2020; Published: 20 February 2020

\begin{abstract}
Steroidogenic Factor 1 (SF-1/NR5A1), an orphan nuclear receptor, is important for sexual differentiation and the development of multiple endocrine organs, as well as cell proliferation in cancer cells. Activating transcription factor 3 (ATF3) is a transcriptional repressor, and its expression is rapidly induced by DNA damage and oncogenic stimuli. Since both NR5A1 and ATF3 can regulate and cooperate with several transcription factors, we hypothesized that NR5A1 may interact with ATF3 and plays a functional role in cancer development. First, we found that NR5A1 physically interacts with ATF3. We further demonstrated that ATF3 expression is up-regulated by NR5A1. Moreover, the promoter activity of the ATF3 is activated by NR5A1 in a dose-dependent manner in several cell lines. By mapping the ATF3 promoter as well as the site-directed mutagenesis analysis, we provide evidence that NR5A1 response elements ( $-695 \mathrm{bp}$ and $-665 \mathrm{bp}$ ) are required for ATF3 expression by NR5A1. It is well known that the transcriptional activities of NR5A1 are modulated by post-translational modifications, such as small ubiquitin-related modifier (SUMO) modification and phosphorylation. Notably, we found that both SUMOylation and phosphorylation of NR5A1 play roles, at least in part, for NR5A1-mediated ATF3 expression. Overall, our results provide the first evidence of a novel relationship between NR5A1 and ATF3.
\end{abstract}

Keywords: NR5A1; ATF3; interaction; transcriptional activity; SUMOylation; phosphorylation

\section{Introduction}

Steroidogenic factor 1 (NR5A1) (also called SF-1) is an orphan nuclear receptor superfamily critical for regulation of sex determination, adrenal and gonadal development, reproductive function, and steroidogenesis [1-4]. Furthermore, recent studies have indicated that NR5A1 is associated with adrenocortical cancer development [5-8]. NR5A1 targets a variety of genes such as CYP17, DAX-1, and STAR [9-13], and regulates transcription of these genes through cooperating with multiple transcription factors and cofactors, including GATA4, SOX9, EGR1, and WT1 [14-17]; however, the molecular mechanisms of NR5A1 in cancer development are still largely unknown.

The majority of the transcription factors are regulated by post-translational modifications which are essential for normal physiological functions in cells. Small ubiquitin-related modifier (SUMO) modification is one type of post-translational modifications, and SUMOylation of transcription factors and nuclear receptors has a strong impact on their transcriptional activities [18-20]. Mounting evidence has demonstrated that NR5A1 can be SUMOylated on lysine 194 (K194, the major site) and lysine 119 (K119, the minor site), and SUMO modification of NR5A1 regulates its transcriptional activities [21-24]. In addition to the SUMOylation, phosphorylation at serine 203 (S203) plays a key in the transcriptional capacity of NR5A1 $[25,26]$. Moreover, several reports have demonstrated that 
NR5A1 activity through phosphorylation at S203 is inhibited by SUMOylation of NR5A1 [27,28], suggesting the important interaction between SUMOylation and phosphorylation. These evidence indicates that post-translational modifications such as phosphorylation and SUMOylation of NR5A1 may affect NR5A1 function in cancer cells.

Activating transcription factor 3 (ATF3), which is a member of the ATF/CREB family of transcriptional repressors, binds the ATF/CAMP response element (CRE) of a number of promoters to regulate its downstream target genes [29]. ATF3 is rapidly induced in cells once exposed to stress stimuli including those initiated by cytokines, genotoxic agents, infections, nerve injury, tissue damage, or physiological stresses $[29,30]$. In addition, a solid line of evidence has indicated that ATF3 is able to suppress cell growth and inhibit the development of tumors [31-33]; however, some evidence has implicated that ATF3 is up-regulated in many cancers, suggesting that ATF3 is an oncogene [34,35]. These results imply that ATF3 expression has positive and negative effects on proliferation and survival of cancer cells.

In the present work, we report the relationship between NR5A1 and ATF3. NR5A1 physically interacts with ATF3, and NR5A1 binds ATF3 promoter to enhance the ATF3 transcriptional activity. Interestingly, we have demonstrated that both phosphorylation and SUMOylation of NR5A1 have functions in NR5A1-mediated ATF3 expression. Taken together, these results support a novel relationship between NR5A1 and ATF3 and this relationship may have an impact in organ differentiation and cancer development.

\section{Results}

\subsection{Steroidogenic Factor 1 (NR5A1) Physically Interacts with Activating Transcription Factor 3 (ATF3)}

Since NR5A1 cooperates with several transcription factors [14,15,36,37], we first analyzed whether NR5A1 can bind to ATF3. To address this question, we co-transfected HIS-FLAG-tagged ATF3 with or without HA-tagged NR5A1 expression plasmids into H1299 cells, which do not express endogenous NR5A1, and $\mathrm{Ni}^{2+}$-bead pull-down assay was performed. As shown in Figure 1, when both ATF3 and NR5A1 were expressed, the NR5A1 band was observed in $\mathrm{Ni}^{2+}$-bead pull-down samples. To further investigate whether the ATF3 physically binds to NR5A1, HIS-FLAG-tagged ATF3 was expressed in the Y1 cells, which endogenously express NR5A1, and $\mathrm{Ni}^{2+}$-bead pull-down assay was performed. As shown in Figure 1, HIS-FLAG-tagged ATF3 was expressed in Y1 cells and was precipitated (WCL and $\mathrm{Ni}^{2+}$-bead pull-down). NR5A1 was also present in the cell lysate (WCL) and co-precipitated with HIS-FLAG-tagged ATF3 $\left(\mathrm{Ni}^{2+}\right.$-bead pull-down). Notably, the endogenous NR5A1 on Y1 cells is smaller than HA-tagged NR5A1 on H1299 cells. These results indicate that NR5A1 physically binds to ATF3.

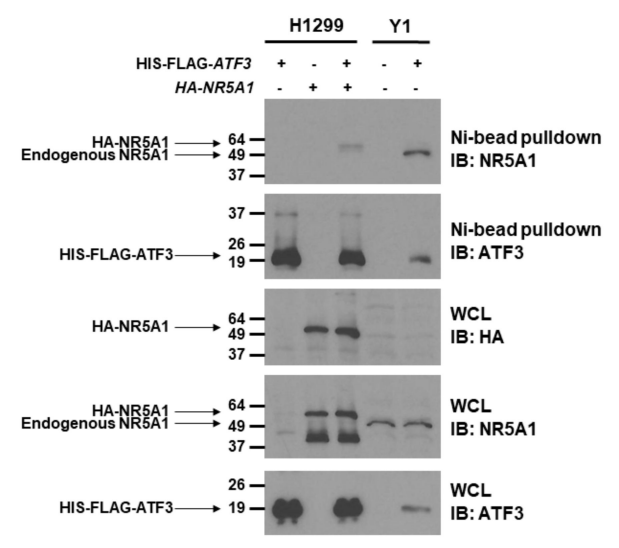

Figure 1. Steroidogenic factor 1 (NR5A1) physically interacts with activating transcription factor 3 (ATF3). H1299 cells were transfected with of HIS-FLAG-tagged ATF3 or HA-tagged NR5A1 or both expression plasmids. Y1 cells were transfected with HIS-FLAG-tagged ATF3 expression plasmid. Forty-eight hours later, the cell lysates were immunoprecipitated by anti-HIS antibody, followed by anti-ATF3 or anti-NR5A1 immunoblotting. WCL indicates whole cell lysates. 


\subsection{NR5A1 Increases ATF3 Protein Level}

Since NR5A1 is a transcription factor and binds to ATF3, we next investigated the role of NR5A1 on ATF3 expression. Expression plasmids encoding wild-type NR5A1 or empty plasmids were transfected into H1299, MCF7, and Y1 cells. As shown in Figure 2, expression of wild-type NR5A1 led to increase ATF3 protein levels in H1299, MCF7, and Y1 cells (approximately 1.8-2.0 fold). This finding indicates that NR5A1 has potential to promote ATF3 expression.

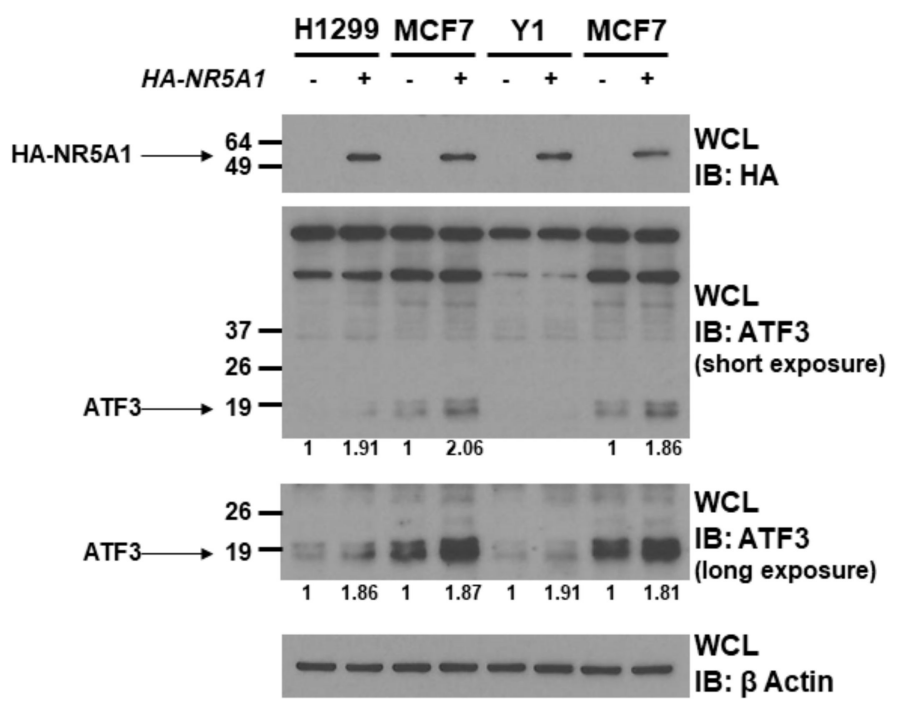

Figure 2. NR5A1 increases ATF3 protein level. H1299, MCF7, and Y1 cells were transfected with either pcDNA3 vector or wild-type HA-tagged NR5A1 expression plasmid. Forty-eight hours later, the expression levels of NR5A1 and ATF3 were determined using anti-HA and anti-ATF3 immunoblotting, respectively. The $\beta$-Actin levels were also determined for equal loading.

\subsection{NR5A1 Is an Activator of the ATF3 Promoter}

As NR5A1 increases ATF3 expression, we next investigated the role of NR5A1 on ATF3 promoter activation. The -1372 bp ATF3 promoter-LUC reporter plasmid was co-transfected with NR5A1 expression plasmid into several different cell lines and ATF3 promoter activity was determined by measuring the LUC activity in cell lysates $48 \mathrm{~h}$ after transfection. As shown in Figure $3 \mathrm{~A}-\mathrm{C}$, expression of NR5A1 generated a dose-dependent increase in the activity of ATF3 gene transcription. This finding indicates that NR5A1 is an activator of the ATF3 transcription. 
A

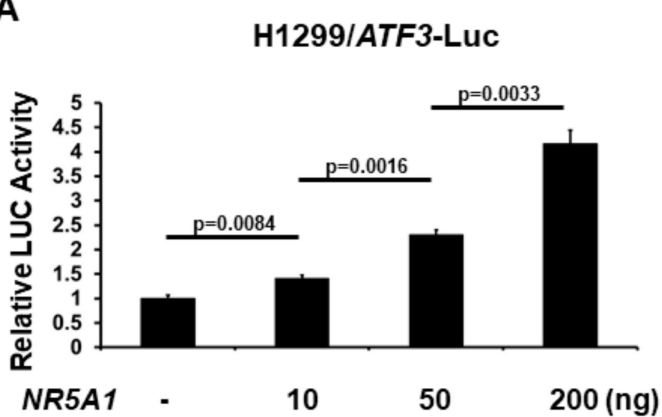

B
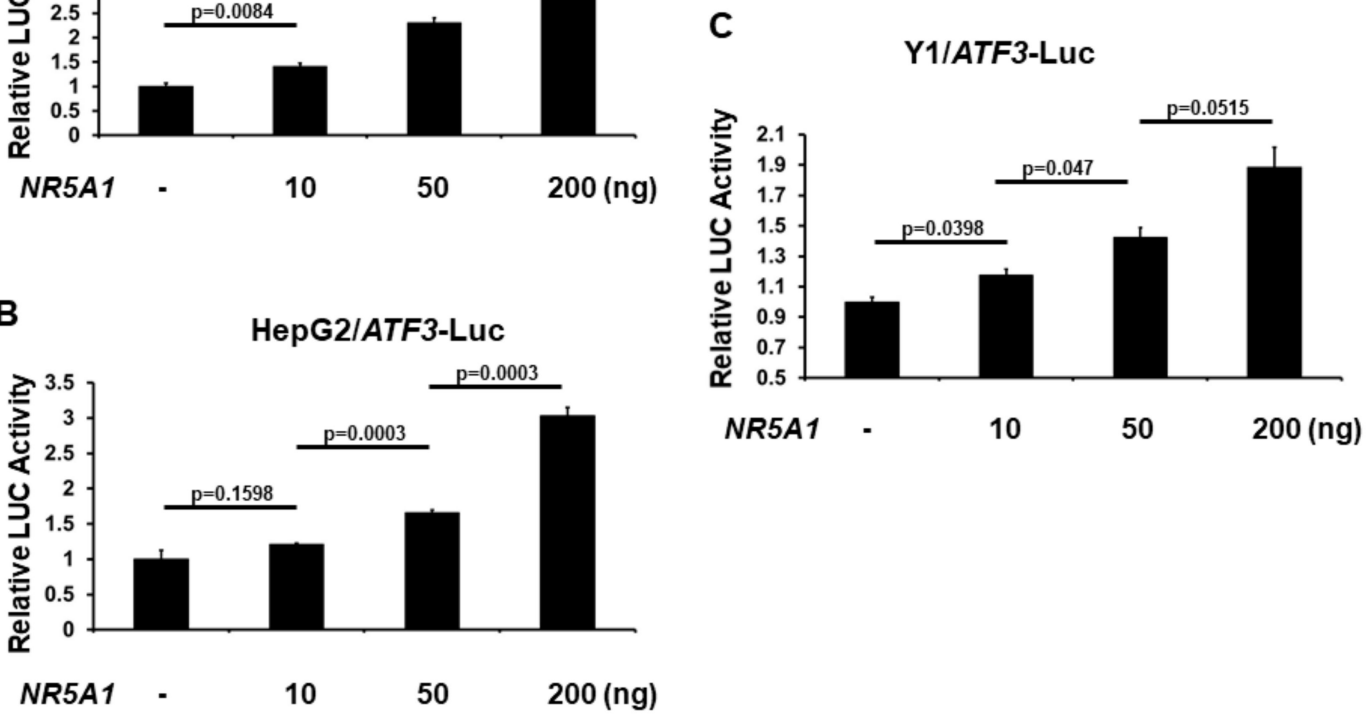

Figure 3. NR5A1 activates ATF3 transcription. (A) H1299, (B) HepG2, and (C) Y1 cells were co-transfected, where indicated, with different amount of NR5A1 expression plasmid and ATF3 promoter-LUC reporter plasmid. Luciferase activities were measured $48 \mathrm{~h}$ after transfection and normalized with Renilla activity. Relative LUC activity was calculated and plotted.

\subsection{Minimal ATF3 Promoter Region Responsive to NR5A1 Activation}

Because the -1372 bp ATF3 promoter contains two potential candidates of NR5A1 response elements (REs), the ATF3 promoter was truncated to determine the minimal region that is important for transcriptional activation by NR5A1 (Figure 4A). Deletion of the distal NR5A1 RE (-695 bp), as shown in the -680 bp promoter, resulted in a slight loss of NR5A1-mediated ATF3 transcriptional activity. However, when both NR5A1 REs were truncated, as shown in the -300 bp promoter, the NR5A1-mediated ATF3 transcriptional activity was significantly decreased (approximately 75\% loss).

To further determine whether the two NR5A1 REs are required for NR5A1-mediated ATF3 expression, we first searching potential NR5A1 binding site(s) on ATF3 promoter region using ALGGEN-PROMO website (http://alggen.lsi.upc.es/cgi-bin/promo_v3/promo/promoinit.cgi?dirDB= TF_8.3). We identified two potential NR5A1 binding sites on the human ATF3 promoter region. The two potential NR5A1 binding sites are located 695 bp (CGCCGCAGAGGTCACACCCGG) and $665 \mathrm{bp}$ (TGACTTTGGACACCTTCCCC) upstream of the transcriptional start site, suggesting that NR5A1 may regulate ATF3 transcription directly. We next generated $-695 \mathrm{bp}$ mutant (CAGAGGTCA $\rightarrow$ CAGAAATCA) and -665 bp mutant (GGACACCTTC $\rightarrow$ GGACAAATTC) ATF3 promoter-LUC reporter plasmids. As shown in Figure 4B, mutation of either $-695 \mathrm{bp}$ or $-665 \mathrm{bp}$ NR5A1 RE resulted in approximately $40 \%$ of NR5A1-mediated ATF3 promoter activity. Notably, mutations of both $-695 \mathrm{bp}$ and $-665 \mathrm{bp}$ NR5A1 REs dramatically reduced ATF3 promoter activity (approximately 70-75\% loss). Together, these results indicate that both $-695 \mathrm{bp}$ and $-665 \mathrm{bp}$ REs are essential for the NR5A1 action on the ATF3 promoter. 
A

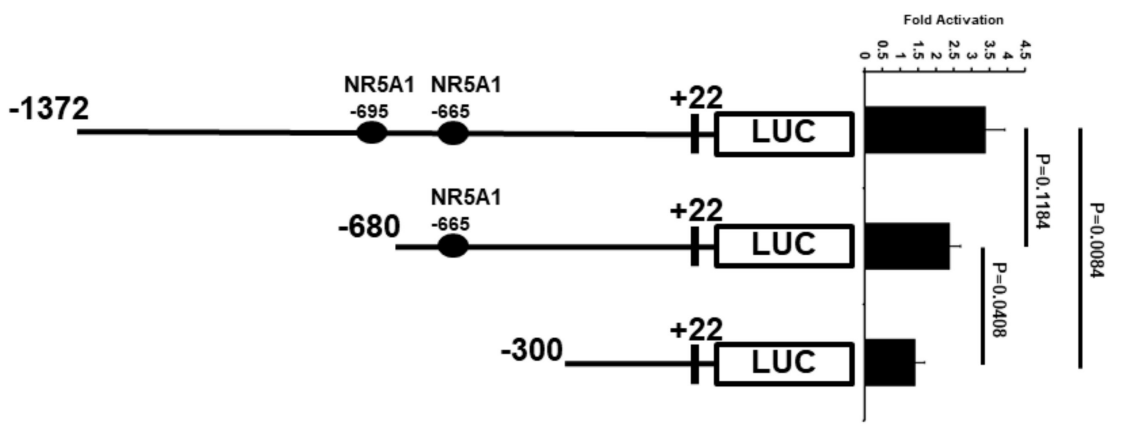

B

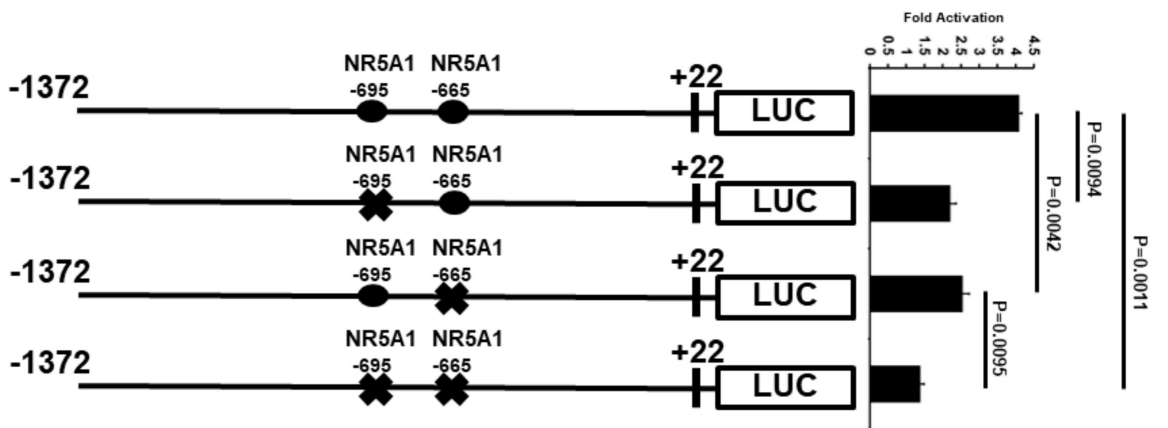

Figure 4. Regions of ATF3 promoter important for transcriptional up-regulation by NR5A1. (A) H1299 cells were co-transfected with ATF3 promoter deletion constructs and NR5A1 expression plasmids. Luciferase activities were measured $48 \mathrm{~h}$ after transfection and normalized with Renilla activity. Relative LUC activity was calculated and plotted. (B) H1299 cells were co-transfected NR5A1 expression plasmids with either -1372 wild-type, -695 RE mutated, -665 RE mutated, or both -695 and -665 REs mutated ATF3 promoter constructs. Luciferase activities were measured $48 \mathrm{~h}$ after transfection and normalized with Renilla activity. Relative LUC activity was calculated and plotted.

2.5. Phosphorylation and Small Ubiquitin-Related Modifier (SUMO)ylation of NR5A1 Are Required for Full NR5A1-Mediated ATF3 Transcriptional Activity

Because NR5A1 has been demonstrated to be modified by post-translational modifications such as phosphorylation and SUMOylation [21-26], we next examined the effect of the post-translational modifications of NR5A1 on its transcriptional activity of the ATF3 promoter. H1299 and Saos2 cells were co-transfected the ATF3 promoter-LUC reporter plasmid with either wild-type (WT), K119R (mimicking de-SUMOylated at K119), K194R (mimicking de-SUMOylated at K194), 2KR (mimicking de-SUMOylated at both K119 and K194), S203A (mimicking de-phosphorylated at S203), or SUMO-WT (mimicking SUMOylated) NR5A1 expression plasmid. As shown in Figure 5A and 5B, in both H1299 and Saos2 cell lines, while the WT and SUMO-WT NR5A1 enhanced ATF3 promoter activity, K119R, K194R, 2KR, and S203A NR5A1 reduced this effect. Interestingly, loss of phosphorylation reduced more NR5A1-mediated ATF3 promoter activity than loss of SUMOylation did, suggesting that phosphorylation is more important for NR5A1 on ATF3 promoter activity. These results suggest that both phosphorylation and SUMOylation are essential, at least in part, for NR5A1-mediated ATF3 promoter activity. 
A

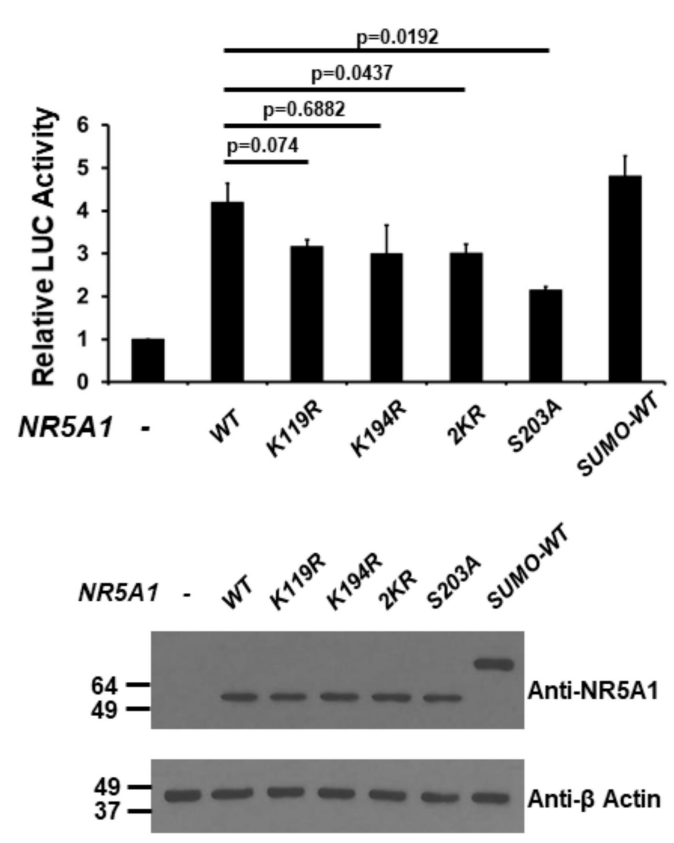

B

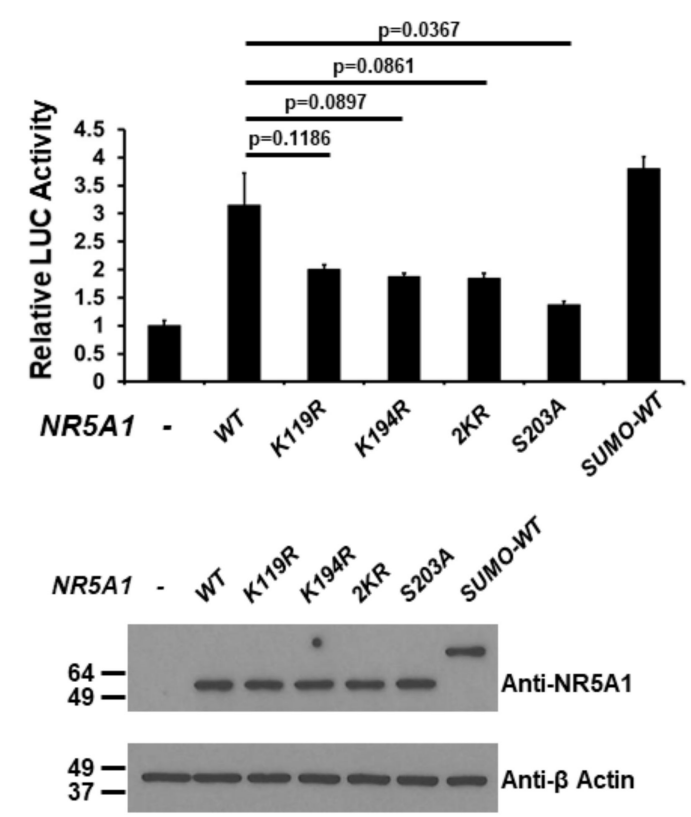

Figure 5. Post-translational modifications of NR5A1 regulate the activation of the ATF3 promoter. (A) H1299 and (B) Saos2 cells were co-transfected with the ATF3 promoter-LUC reporter plasmid and either wild-type (WT), K119R, K194R, 2KR, S203A, or small ubiquitin-related modifier (SUMO)-WT NR5A1 expression plasmid. Luciferase activities were measured $48 \mathrm{~h}$ after transfection and normalized with Renilla activity. Relative LUC activity was calculated and plotted.

\section{Discussion}

The growth and development of tissue and organisms requires precisely orchestrated gene regulation and cell proliferation. NR5A1, an orphan member of the nuclear receptor superfamily, is expressed in the steroidogenic adrenals and gonads specifically $[4,38,39]$. Although NR5A1 has numerous functions including sex determination, adrenal development, and steroidogenesis [1-4], it is also involved in cancer development [5-8]. Mutations of NR5A1 lead to many disorders and phenotypes, including premature ovarian failure, adrenocortical insufficiency, sex reversal, and spermatogenesis failure. Herein, we demonstrate a novel NR5A1 and ATF3 interaction, and post-translational modifications such as phosphorylation and SUMOylation are involved in this relationship between NR5A1 and ATF3.

NR5A1 can cooperate with several transcription factors [14-17], and in the present study, we observed the physical interaction of NR5A1 with ATF3. Another AP-1 protein family member, JDP2, also binds to NR5A1 (data not shown), suggesting that the members of AP-1 protein family may have potential binding to NR5A1. NR5A1 is overexpressed in adrenocortical tumors [5-7], suggesting that NR5A1 relates to cancer development. Moreover, ATF3 is induced by stress stimuli and associates with various kinds of cancer development [29,30,34]. Previous reports have demonstrated that ATF3 is expressed in H295R human adrenal carcinoma cells [40] and may be involved in adrenocortical aldosterone synthesis [41]. In addition, co-transfection of ATF3 and a member of the AP-1 complex JUNB into H295R cells increases STAR expression, which is also a downstream target of NR5A1 [13,40]. Taken together, NR5A1-ATF3 interaction and axis may regulate cell population in adrenocortical cancer cells through regulation of cell proliferation related genes. However, whether NR5A1-ATF3 interaction regulates NR5A1-mediated ATF3 expression in a feedback loop remains unknown. Further future studies are indeed necessary to dissect whether NR5A1-ATF3 axis directly regulates aldosterone synthesis in adrenals and is directly involved in cancer development. 
It is well known that NR5A1, as a critical master regulator, helps control the activities of numerous genes related to the development of the adrenals and the ovaries (testes and ovaries) [9-13]. In the present work, we showed that NR5A1 up-regulates ATF3 protein level and enhances ATF3 promoter activity. Our promoter analysis further supports that both NR5A1 response elements $-695 \mathrm{bp}$ and $-665 \mathrm{bp}$ on the ATF3 promoter region are essential for regulating ATF3 gene expression. Our data highlights that ATF3 is the novel target gene for NR5A1.

Post-translational modifications such as phosphorylation, SUMOylation, and acetylation influence wide range of cellular activities, including cancer development [42,43]. It has been determined that NR5A1 can be SUMOylated on K194 and K119, which are major and minor sites, respectively, and the SUMOylation of NR5A1 influences its transcriptional activities [21-24]. In the present work, we demonstrated that replacement of K194 and/or K119 by an arginine residue in NR5A1 leads to reduce ATF3's transcriptional activity, suggesting that SUMOylation of NR5A1 plays an important role for ATF3 expression. Moreover, as a transcription factor, NR5A1 has been shown to be phosphorylated at S203 [25,26]. Importantly, loss of phosphorylation on NR5A1 S203 (S203A) significantly decreases its activity in regulating the ATF3 promoter more than SUMOylation mutants. This result, consistent with previous reports, further highlights the crucial role of phosphorylation on NR5A1 function as a transcription factor. The acetylation of NR5A1 has also been studied extensively. The major acetylation sites on NR5A1 based on reports are K34, K38, and K72 [44] and amino acids from 56-63 and 102-106 [45]. Overall, we provide the first evidence that phosphorylation and SUMOylation of NR5A1 play an important function in regulation of ATF3 gene activity.

In conclusion, this investigation demonstrated that NR5A1 physically interacts with ATF3. Our study also identified that NR5A1 is a novel activator of ATF3 promoter, and that the post-translational modifications (phosphorylation and SUMOylation) play a critical role for NR5A1's transcriptional activity.

\section{Materials and Methods}

\subsection{Chemicals and Reagents}

Cell culture media and reagents were purchased from Thermo Fisher Scientific (Waltham, MA, USA). Antibodies against ATF3 and $\beta$-Actin (Santa Cruz Biotechnology Inc., Santa Cruz, CA, USA), HIS (Origene, Rockville, MD, USA) and NR5A1 (Upstate Biochemistry Inc., Charlottsville, VA, USA) were purchased commercially. Luciferase activity was measured using the Dual-Luciferase Reporter Assay System (Promega, Madison, WI, USA).

\subsection{DNA Constructs}

HIS-FLAG NR5A1-pcDNA3 expression plasmid was generated by PCR cloning and described previously [21]. S203A, K119R, K194R, and 2KR NR5A1 expression plasmids were created by PCR-based mutagenesis (QuikChange Lightning site-directed mutagenesis kit, Agilent/Strategene, La Jolla, CA, USA). SUMO-WT NR5A1 expression plasmid was created by inserting SUMO1 DNA sequence into the N-terminal NR5A1 DNA sequence. The human ATF3 promoter (-1372/+22 bp) pGL2 plasmid was kindly provided by Dr. Aronheim (Technion-Israel Institute of Technology, Haifa, Israel). The human ATF3 promoter deletion constructs were then generated by removal of specific fragments of DNA sequence in Yang lab. The human ATF3 promoter with NR5A1 RE mutant plasmids were created by PCR-based mutagenesis (QuikChange Lightning site-directed mutagenesis kit, Agilent/Strategene, La Jolla, CA, USA). All constructs were verified by Sanger nucleotide sequencing.

\subsection{Cell Culture and Transfection}

H1299, HepG2, MCF7, Y1, and Saos2 cells were obtained from the American Type Culture Collection (Manassas, VA, USA). The cells were maintained in Dulbecco's-modified Eagle medium (DMEM) in the presence of 10\% fetal bovine serum and Pen/Strep antibiotics (GIBCO/Life Technologies, 
Grand Island, NY, USA) in humidified air containing $5 \% \mathrm{CO}_{2}$ at $37{ }^{\circ} \mathrm{C}$ and cultured for less than six months. After incubation, the cells were transfected with specific expression plasmids described in each assay using Fugene HD Transfection Reagent (Roche, Madison, WI, USA). Forty-eight hours after transfection, the cells were harvested and lysed.

\subsection{ATF3 Promoter luciferase Reporter Assays}

Cells were cultured in 24-well plates overnight and then transiently transfected with ATF3 promoter-firefly luciferase plasmid and internal control pRL-TK plasmid (which encodes Renilla luciferase activity) in the presence of Fugene HD Transfection Reagent (Roche, Madison, WI, USA). At $48 \mathrm{~h}$ after transfection, the cells were harvested and lysed in passive lysis buffer (Promega). Luminescence was detected with the Dual-Luciferase Reporter Assay System (Promega) according to the manufacturer's instructions. Firefly luciferase activity was normalized by calculating the ratio to Renilla luciferase activity. The relative luciferase activity was calculated as a fold change to the control groups. All experiments were performed three times in triplicate setting.

\subsection{Immunoprecipitation Assays}

Y1, MCF7, and H1299 cells $\left(2 \times 10^{6}\right)$ were seeded onto $100 \mathrm{~mm}$ plates. Forty-eight hours after transfection, cells were harvested and lysed in Sabatini lysis buffer (40 mM HEPES, $120 \mathrm{mM}$ sodium chloride, $10 \mathrm{mM}$ sodium glycerophosphate, $10 \mathrm{mM}$ sodium pyrophosphate, $50 \mathrm{mM}$ sodium fluoride, $0.5 \mathrm{mM}$ sodium orthovanadate, $1 \mathrm{mM}$ EDTA, $1 \%$ Triton X-100) containing protease inhibitor cocktail (Sigma, St. Louis, MO, USA), followed by rotation for $1 \mathrm{~h}$ at $4{ }^{\circ} \mathrm{C}$ to solubilize proteins. Soluble proteins were collected and immunoprecipitated with the indicated antibody overnight. Protein A agarose beads were added to protein lysates for $2 \mathrm{~h}$ at $4{ }^{\circ} \mathrm{C}$. Beads were centrifuged and washed at least three times with lysis buffer. For $\mathrm{Ni}^{2+}$-bead pull-down assays, $\mathrm{Ni}^{2+}$-NTA agarose was used to precipitate HIS-tagged ATF3 or HIS-tagged NR5A1 from cell lysates. Proteins were eluted by boiling in $40 \mu \mathrm{L}$ of $2 \times$ Laemmli sample buffer, resolved by $8-10 \%$ sodium dodecyl sulfate-polyacrylamide gel electrophoresis (SDS-PAGE), and processed for immunoblotting as described below.

\subsection{Western Blot Analysis}

Protein lysates were allowed to rotate at $4{ }^{\circ} \mathrm{C}$ for $1 \mathrm{~h}$, and protein contents of the high-speed supernatant were determined using the $\mathrm{BCA}^{\mathrm{TM}}$ Protein Assay kit assay (Pierced/Thermo Scientific, Rockford, IL, USA). Equivalent quantities of protein $(40 \mu \mathrm{g})$ were resolved on polyacrylamide-SDS gels, transferred to polyvinylidene difluoride (PVDF) membrane (Bio-Rad, Hercules, CA, USA), and immunoblotted with specific antibodies. Results were visualized using the Supersignal West Dura Extended Duration Substrate kit (Pierce Chemical Co., Rockford, IL, USA). The intensity of the protein band was quantified by ImageJ program.

\subsection{Statistical Analysis}

We performed statistical analysis for the significance of the differences between two measurements by using the Student's $t$ test. $p<0.05$ was considered statistically significant between groups.

\section{Conclusions}

In summary, we have shown a novel relationship between NR5A1 and ATF3 for the first time. Our studies suggest the possibility of interaction between NR5A1 and ATF3 associated with cell proliferation in adrenocortical cancer cells. Moreover, NR5A1 is a novel activator of the ATF3 promoter, and the NR5A1-mediated ATF3 transcription is, at least in part, regulated by the post-translational modifications (phosphorylation and SUMOylation). Together, our findings add a new layer of information to the previous understanding of how NR5A1 functions in steroidogenesis, reproductive organ differentiation, and even cancer development. 
Author Contributions: Conception and design: N.E. and W.-H.Y.; Analysis and interpretation of data: N.E., C.-M.W., W.H.Y., and W.-H.Y.; Development of methodology: N.E., C.-M.W., William H.Y. and W.-H.Y.; Writing, review, and/or revision of the manuscript: N.E., C.-M.W., William H.Y., and W.-H.Y. All authors have read and agreed to the published version of the manuscript.

Funding: This work was supported by grants from a Mercer University Seed Grant (W.-H.Y.), a Rubye Smith Research Grant (W.-H.Y.) and a Larsen Research Endowment Fellowship Program Grant (W.-H.Y.).

Conflicts of Interest: The authors declare no conflict of interest.

\section{Abbreviations}

NR5A1 Steroidogenic factor 1 (SF-1)

ATF3 Activating transcription factor 3

SUMO small ubiquitin-like modifier

\section{References}

1. Luo, X.; Ikeda, Y.; Parker, K.L. A cell-specific nuclear receptor is essential for adrenal and gonadal development and sexual differentiation. Cell 1994, 77, 481-490. [CrossRef]

2. Wong, M.; Ikeda, Y.; Luo, X.; Caron, K.M.; Weber, T.J.; Swain, A.; Schimmer, B.P.; Parker, K.L. Steroidogenic factor 1 plays multiple roles in endocrine development and function. Recent Prog. Horm. Res. 1997, 52, 167-182.

3. Hammer, G.D.; Ingraham, H.A. Steroidogenic factor-1: Its role in endocrine organ development and differentiation. Front. Neuroendocrinol. 1999, 20, 199-223. [CrossRef]

4. Val, P.; Lefrancois-Martinez, A.M.; Veyssiere, G.; Martinez, A. SF-1 a key player in the development and differentiation of steroidogenic tissues. Nucl. Recept. 2003, 1, 8. [CrossRef] [PubMed]

5. Almeida, M.Q.; Soares, I.C.; Ribeiro, T.C.; Fragoso, M.C.; Marins, L.V.; Wakamatsu, A.; Ressio, R.A.; Nishi, M.Y.; Jorge, A.A.; Lerario, A.M.; et al. Steroidogenic factor 1 overexpression and gene amplification are more frequent in adrenocortical tumors from children than from adults. J. Clin. Endocrinol. Metab. 2010, 95, 1458-1462. [CrossRef] [PubMed]

6. Figueiredo, B.C.; Cavalli, L.R.; Pianovski, M.A.; Lalli, E.; Sandrini, R.; Ribeiro, R.C.; Zambetti, G.; DeLacerda, L.; Rodrigues, G.A.; Haddad, B.R. Amplification of the steroidogenic factor 1 gene in childhood adrenocortical tumors. J. Clin. Endocrinol. Metab. 2005, 90, 615-619. [CrossRef]

7. Pianovski, M.A.; Cavalli, L.R.; Figueiredo, B.C.; Santos, S.C.; Doghman, M.; Ribeiro, R.C.; Oliveira, A.G.; Michalkiewicz, E.; Rodrigues, G.A.; Zambetti, G.; et al. SF-1 overexpression in childhood adrenocortical tumours. Eur. J. Cancer 2006, 42, 1040-1043. [CrossRef] [PubMed]

8. Doghman, M.; Karpova, T.; Rodrigues, G.A.; Arhatte, M.; De Moura, J.; Cavalli, L.R.; Virolle, V.; Barbry, P.; Zambetti, G.P.; Figueiredo, B.C.; et al. Increased steroidogenic factor-1 dosage triggers adrenocortical cell proliferation and cancer. Mol. Endocrinol. 2007, 21, 2968-2987. [CrossRef]

9. Dammer, E.B.; Leon, A.; Sewer, M.B. Coregulator exchange and sphingosine-sensitive cooperativity of steroidogenic factor-1, general control nonderepressed 5, p54, and p160 coactivators regulate cyclic adenosine 3',5'-monophosphate-dependent cytochrome P450c17 transcription rate. Mol. Endocrinol. 2007, 21, 415-438. [CrossRef]

10. Zhang, P.; Mellon, S.H. The orphan nuclear receptor steroidogenic factor-1 regulates the cyclic adenosine 3', 5'-monophosphate-mediated transcriptional activation of rat cytochrome P450c17 (17 alpha-hydroxylase/c17-20 lyase). Mol. Endocrinol. 1996, 10, 147-158.

11. Burris, T.P.; Guo, W.; Le, T.; McCabe, E.R. Identification of a putative steroidogenic factor-1 response element in the DAX-1 promoter. Biochem. Biophys. Res. Commun. 1995, 214, 576-581. [CrossRef] [PubMed]

12. Kawabe, K.; Shikayama, T.; Tsuboi, H.; Oka, S.; Oba, K.; Yanase, T.; Nawata, H.; Morohashi, K. Dax-1 as one of the target genes of Ad4BP/SF-1. Mol. Endocrinol. 1999, 13, 1267-1284. [CrossRef]

13. Sugawara, T.; Kiriakidou, M.; McAllister, J.M.; Holt, J.A.; Arakane, F.; Strauss, J.F., 3rd. Regulation of expression of the steroidogenic acute regulatory protein (StAR) gene: A central role for steroidogenic factor 1. Steroids 1997, 62, 5-9. [CrossRef] 
14. Tremblay, J.J.; Viger, R.S. Transcription factor GATA-4 enhances Mullerian inhibiting substance gene transcription through a direct interaction with the nuclear receptor SF-1. Mol. Endocrinol. 1999, 13, 1388-1401. [PubMed]

15. Dorn, C.; Ou, Q.; Svaren, J.; Crawford, P.A.; Sadovsky, Y. Activation of luteinizing hormone beta gene by gonadotropin-releasing hormone requires the synergy of early growth response-1 and steroidogenic factor-1. J. Biol. Chem. 1999, 274, 13870-13876. [CrossRef] [PubMed]

16. Wilson, M.J.; Jeyasuria, P.; Parker, K.L.; Koopman, P. The transcription factors steroidogenic factor-1 and SOX9 regulate expression of Vanin-1 during mouse testis development. J. Biol. Chem. 2005, 280, 5917-5923. [CrossRef] [PubMed]

17. Gurates, B.; Amsterdam, A.; Tamura, M.; Yang, S.; Zhou, J.; Fang, Z.; Amin, S.; Sebastian, S.; Bulun, S.E. WT1 and DAX-1 regulate SF-1-mediated human P450arom gene expression in gonadal cells. Mol. Cell. Endocrinol. 2003, 208, 61-75. [CrossRef]

18. Gong, Z.; Brackertz, M.; Renkawitz, R. SUMO modification enhances p66-mediated transcriptional repression of the Mi-2/NuRD complex. Mol. Cell. Biol. 2006, 26, 4519-4528. [CrossRef]

19. Rytinki, M.M.; Palvimo, J.J. SUMOylation modulates the transcription repressor function of RIP140. J. Biol. Chem. 2008, 283, 11586-11595. [CrossRef]

20. Duverger, O.; Chen, S.X.; Lee, D.; Li, T.; Chock, P.B.; Morasso, M.I. SUMOylation of DLX3 by SUMO1 promotes its transcriptional activity. J Cell. Biochem. 2011, 112, 445-452. [CrossRef]

21. Wang, C.M.; Liu, R.; Wang, L.; Yang, W.H. Acidic residue Glu199 increases SUMOylation level of nuclear hormone receptor NR5A1. Int. J. Mol. Sci. 2013, 14, 22331-22345. [CrossRef]

22. Chen, W.Y.; Lee, W.C.; Hsu, N.C.; Huang, F.; Chung, B.C. SUMO modification of repression domains modulates function of nuclear receptor 5A1 (steroidogenic factor-1). J. Biol. Chem. 2004, 279, 38730-38735. [CrossRef]

23. Komatsu, T.; Mizusaki, H.; Mukai, T.; Ogawa, H.; Baba, D.; Shirakawa, M.; Hatakeyama, S.; Nakayama, K.I.; Yamamoto, H.; Kikuchi, A.; et al. Small ubiquitin-like modifier 1 (SUMO-1) modification of the synergy control motif of Ad4 binding protein/steroidogenic factor 1 (Ad4BP/SF-1) regulates synergistic transcription between Ad4BP/SF-1 and Sox9. Mol. Endocrinol. 2004, 18, 2451-2462. [CrossRef] [PubMed]

24. Lee, M.B.; Lebedeva, L.A.; Suzawa, M.; Wadekar, S.A.; Desclozeaux, M.; Ingraham, H.A. The DEAD-box protein DP103 (Ddx20 or Gemin-3) represses orphan nuclear receptor activity via SUMO modification. Mol. Cell. Biol. 2005, 25, 1879-1890. [CrossRef]

25. Hammer, G.D.; Krylova, I.; Zhang, Y.; Darimont, B.D.; Simpson, K.; Weigel, N.L.; Ingraham, H.A. Phosphorylation of the nuclear receptor SF-1 modulates cofactor recruitment: Integration of hormone signaling in reproduction and stress. Mol. Cell. 1999, 3, 521-526. [CrossRef]

26. Lewis, A.E.; Rusten, M.; Hoivik, E.A.; Vikse, E.L.; Hansson, M.L.; Wallberg, A.E.; Bakke, M. Phosphorylation of steroidogenic factor 1 is mediated by cyclin-dependent kinase 7. Mol. Endocrinol. 2008, 22, 91-104. [CrossRef] [PubMed]

27. Yang, W.H.; Heaton, J.H.; Brevig, H.; Mukherjee, S.; Iniguez-Lluhi, J.A.; Hammer, G.D. SUMOylation inhibits SF-1 activity by reducing CDK7-mediated serine 203 phosphorylation. Mol. Cell. Biol. 2009, 29, 613-625. [CrossRef] [PubMed]

28. Campbell, L.A.; Faivre, E.J.; Show, M.D.; Ingraham, J.G.; Flinders, J.; Gross, J.D.; Ingraham, H.A. Decreased recognition of SUMO-sensitive target genes following modification of SF-1 (NR5A1). Mol. Cell. Biol. 2008, 28, 7476-7486. [CrossRef]

29. Hai, T.; Hartman, M.G. The molecular biology and nomenclature of the activating transcription factor/cAMP responsive element binding family of transcription factors: Activating transcription factor proteins and homeostasis. Gene 2001, 273, 1-11. [CrossRef]

30. Hai, T.; Wolfgang, C.D.; Marsee, D.K.; Allen, A.E.; Sivaprasad, U. ATF3 and stress responses. Gene Expr. 1999, 7, 321-335.

31. Lu, D.; Wolfgang, C.D.; Hai, T. Activating transcription factor 3, a stress-inducible gene, suppresses Ras-stimulated tumorigenesis. J. Biol. Chem. 2006, 281, 10473-10481. [CrossRef] [PubMed]

32. Huang, X.; Li, X.; Guo, B. KLF6 induces apoptosis in prostate cancer cells through up-regulation of ATF3. J. Biol. Chem. 2008, 283, 29795-29801. [CrossRef] [PubMed] 
33. Fan, F.; Jin, S.; Amundson, S.A.; Tong, T.; Fan, W.; Zhao, H.; Zhu, X.; Mazzacurati, L.; Li, X.; Petrik, K.L.; et al. ATF3 induction following DNA damage is regulated by distinct signaling pathways and over-expression of ATF3 protein suppresses cells growth. Oncogene 2002, 21, 7488-7496. [CrossRef] [PubMed]

34. Yin, X.; Dewille, J.W.; Hai, T. A potential dichotomous role of ATF3, an adaptive-response gene, in cancer development. Oncogene 2008, 27, 2118-2127. [CrossRef] [PubMed]

35. Pelzer, A.E.; Bektic, J.; Haag, P.; Berger, A.P.; Pycha, A.; Schafer, G.; Rogatsch, H.; Horninger, W.; Bartsch, G.; Klocker, H. The expression of transcription factor activating transcription factor 3 in the human prostate and its regulation by androgen in prostate cancer. J. Urol. 2006, 175, 1517-1522. [CrossRef]

36. Hossain, A.; Saunders, G.F. Role of Wilms tumor 1 (WT1) in the transcriptional regulation of the Mullerian-inhibiting substance promoter. Biol. Reprod. 2003, 69, 1808-1814. [CrossRef]

37. De Santa Barbara, P.; Bonneaud, N.; Boizet, B.; Desclozeaux, M.; Moniot, B.; Sudbeck, P.; Scherer, G.; Poulat, F.; Berta, P. Direct interaction of SRY-related protein SOX9 and steroidogenic factor 1 regulates transcription of the human anti-Mullerian hormone gene. Mol. Cell. Biol. 1998, 18, 6653-6665. [CrossRef]

38. Hatano, O.; Takayama, K.; Imai, T.; Waterman, M.R.; Takakusu, A.; Omura, T.; Morohashi, K. Sex-dependent expression of a transcription factor, Ad4BP, regulating steroidogenic P-450 genes in the gonads during prenatal and postnatal rat development. Development 1994, 120, 2787-2797.

39. Hoivik, E.A.; Lewis, A.E.; Aumo, L.; Bakke, M. Molecular aspects of steroidogenic factor 1 (SF-1). Mol. Cell. Endocrinol. 2010, 315, 27-39. [CrossRef]

40. Nogueira, E.F.; Xing, Y.; Morris, C.A.; Rainey, W.E. Role of angiotensin II-induced rapid response genes in the regulation of enzymes needed for aldosterone synthesis. J. Mol. Endocrinol. 2009, 42, 319-330. [CrossRef]

41. Felizola, S.J.; Nakamura, Y.; Ozawa, Y.; Ono, Y.; Morimoto, R.; Midorikawa, S.; Suzuki, S.; Satoh, F.; Sasano, H. Activating transcription factor 3 (ATF3) in the human adrenal cortex: Its possible involvement in aldosterone biosynthesis. Tohoku J. Exp. Med. 2014, 234, 249-254. [CrossRef] [PubMed]

42. Mukherjee, S.; Cruz-Rodriguez, O.; Bolton, E.; Iniguez-Lluhi, J.A. The in vivo role of androgen receptor SUMOylation as revealed by androgen insensitivity syndrome and prostate cancer mutations targeting the proline/glycine residues of synergy control motifs. J. Biol. Chem. 2012, 287, 31195-31206. [CrossRef] [PubMed]

43. Kubota, Y.; O'Grady, P.; Saito, H.; Takekawa, M. Oncogenic Ras abrogates MEK SUMOylation that suppresses the ERK pathway and cell transformation. Nat. Cell Biol. 2011, 13, 282-291. [CrossRef] [PubMed]

44. Jacob, A.L.; Lund, J.; Martinez, P.; Hedin, L. Acetylation of steroidogenic factor 1 protein regulates its transcriptional activity and recruits the coactivator GCN5. J. Biol. Chem. 2001, 276, 37659-37664. [CrossRef] [PubMed]

45. Chen, W.Y.; Juan, L.J.; Chung, B.C. SF-1 (nuclear receptor 5A1) activity is activated by cyclic AMP via p300-mediated recruitment to active foci, acetylation, and increased DNA binding. Mol. Cell. Biol. 2005, 25, 10442-10453. [CrossRef] [PubMed]

(C) 2020 by the authors. Licensee MDPI, Basel, Switzerland. This article is an open access article distributed under the terms and conditions of the Creative Commons Attribution (CC BY) license (http://creativecommons.org/licenses/by/4.0/). 